\title{
Avaliação do processamento a baixas temperaturas do óleo de fígado de Alaska pollock (Theragra chalcogramma)
}

\author{
Evaluation of low temperature rendering of \\ Alaska pollock (Theragra chalcogramma) liver oil
}

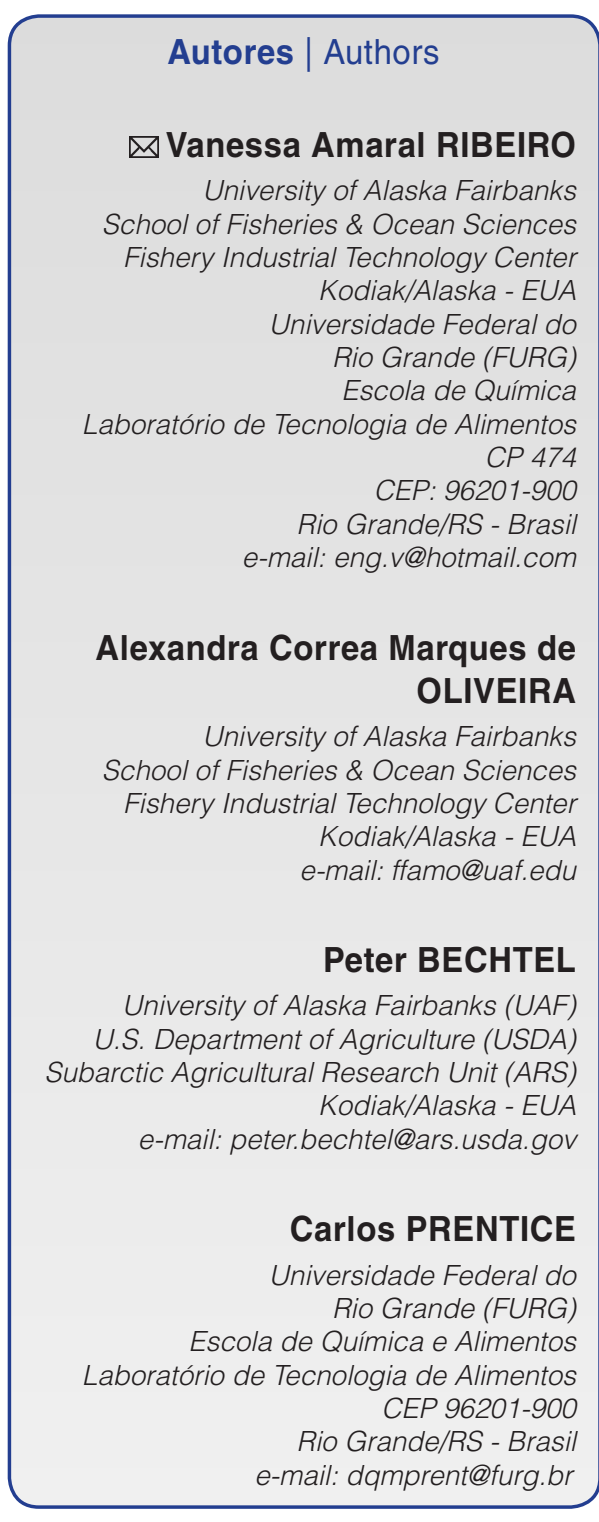

Autor Correspondente / Corresponding Author

Recebido / Received: 27/10/2011 Aprovado / Approved: 04/03/2013 Publicado / Published: jun./2013

\section{Resumo}

O interesse pela produção de óleo de pescado para consumo humano é crescente. No Alasca, EUA, uma grande quantidade de subprodutos tem sido usada como matéria-prima. Entre os subprodutos, o fígado de Alaska pollock (Theragra chalcogramma) apresenta interesse particular em razão de seu alto teor de lipídios. Normalmente, a temperatura usada para extração do óleo de pescado é elevada e potencialmente incompatível com a estabilidade lipídica. O objetivo deste trabalho foi avaliar o processamento do óleo de fígado de Alaska pollock, extraído a baixas temperaturas. Como confirmado, a matéria-prima obtida na primavera (40 \%) apresentou um conteúdo lipídico menor que a de outono (50\%). A extração foi realizada cinco vezes para quatro combinações de tempo (15 e 30 minutos) e temperatura $\left(50\right.$ e $60^{\circ} \mathrm{C}$ ). Foram analisadas duas bateladas de óleo de fígado e vísceras de pollock, produzido em navio processador. O rendimento do processo foi semelhante (aproximadamente $52 \%$ ), os óleos de fígado produzidos se apresentaram mais claros, com coloração amarela, comparados aos de vísceras de pollock produzidos no outono. Os ácidos graxos livres foram similares para óleos de fígado e de vísceras nas duas estações, atingindo, no máximo, 0,4\%; o índice de peróxidos, o valor de anisidina e o valor de ácido tiobarbitúrico determinados nos óleos apresentaram 2-14 meq. $\mathrm{kg}^{-1}$, 20-26 e 0,4-0,6 mg dialdeído malônico. $\mathrm{kg}^{-1}$, respectivamente. Todos os óleos investigados foram compostos principalmente por triacilgliceróis (88-100 \%), com o conteúdo de fósforo não excedendo, em média, 15 ppm.

Palavras-chave: Alaska pollock; Fígado; Óleo; Extração; Valor de ácido Tiobarbitúrico.

\section{Summary}

The demand for edible fish oils is growing. In Alaska, USA, large quantities of fishery byproducts are being used as raw materials, amongst which, Alaska pollock (Theragra chalcogramma) livers are of particular interest due to their high lipid contents. The temperature normally used for the extraction of fish oils is high and potentially incompatible with lipid stability. The goal of this research was to evaluate the processing of Alaska pollock liver oil, processed at low temperatures. As expected, the raw material obtained in the spring ( $40 \%)$ had significantly less lipids than that obtained in the autumn (50\%). The liver oils were rendered five times for each of four combinations of time ( 15 and 30 minutes) and temperature (50 and $60{ }^{\circ} \mathrm{C}$ ). Two batches of liver and viscera pollock oils produced on a processing ship were analyzed. The process yields were similar ( 52\%), and in general, the liver oils produced were lighter and more yellowish in colour than those produced from the pollock viscera in the autumn. The free fatty acid levels were similar for the viscera and liver oils produced in both seasons, reaching a maximum of $0.4 \%$, while the peroxide values, anisidine values and thiobarbituric acid values determined for all the oils were 2-14 meq. $\mathrm{kg}^{-1}, 20-26$ and 0.4-0.6 mg malonic dialdehyde. $\mathrm{kg}^{-1}$, respectively. All the oils investigated were composed mainly of triacylglycerols (88-100\%), with the phosphorus content not exceeding, on average, 15 ppm.

Key words: Alaska Pollock; Liver; Oil; Extraction; TBA value. 


\section{Introdução}

O interesse pelos óleos marinhos destinados ao consumo humano é crescente (BECHTEL, 2007), pois estes são considerados fontes importantes de ácidos graxos poli-insaturados, com ênfase no AEP (ácido eicosapentaenoico 20:5 $\omega-3$ ) e no ADH (ácido docosahexaenoico 22:6 $\omega-3$ ) (FERDOSH et al., 2010), que contribuem para a prevenção de doenças cardiovasculantes (JACHAMANIÁN et al., 2007). O fracionamento físico e a extração com solvente ou com fluidos supercríticos são opções utilizáveis para posterior prensagem e centrifugação (Chantachum et al., 2000), normalmente empregando-se temperaturas de 95 e $100{ }^{\circ} \mathrm{C}$, durante 15 a 20 minutos. O uso de altas temperaturas no processo pode levar a danos oxidativos indesejáveis do óleo obtido (OKADA; MORRISSEY, 2007).

Alaska pollock (Theragra chalcogramma) representa individualmente o maior volume de pesca comercial no mundo, com mais de 3 milhões de toneladas (MCS, 2012). A combinação entre volume e disponibilidade torna interessantes os subprodutos de pollock como matériaprima para a produção de óleo para consumo humano, com 14,3\% de lipídios contidos nas vísceras e 1,2 \% na cabeça (OLIVEIRA e BECHTEL, 2005, 2006). Entre os subprodutos, o fígado é de particular interesse pelo seu teor lipídico - podendo alcançar $50 \%$, dependendo de condições bioecológicas - e também pelo fato de ser facilmente separado dos demais componentes viscerais (BECHTEL e OLIVEIRA, 2006; OLIVEIRA et al., 2008; RIBEIRO, 2010). O objetivo deste trabalho foi avaliar o processamento do óleo de fígado de Alaska pollock extraído a baixas temperaturas.

\section{Material e métodos}

\subsection{Material}

As amostras consistiram de fígados congelados in natura, a $-20 \pm 2{ }^{\circ} \mathrm{C}$, provenientes da espécie Alaska pollock (Theragra chalcogramma) capturada no Mar de Behring, norte do Oceano Pacífico, durante as estações de outono e primavera. O fígado in natura foi separado das vísceras do pollock e processado imediatamente após a captura a bordo, sendo armazenado em blocos congelados até a extração do óleo. Também foi utilizado óleo de fígado produzido no outono e óleo de vísceras processado no outono e na primavera, provenientes da mesma espécie, a bordo do navio American Triumph no Mar de Behring, EUA.

\subsection{Metodologia}

\subsubsection{Processo de extração do óleo}

O processo de extração do óleo em laboratório correspondeu ao que foi proposto no caso do catfish, segundo Sathivel et al. (2003). Foi empregado $(n=5)$ usando-se quatro combinações de tempo e temperatura, a partir de $30 \mathrm{~kg}$ da matéria-prima descongelada e homogeneizada em misturador (Waring modelo CB15, EUA) por 2 minutos a $21.000 \mathrm{rpm}$. O produto obtido foi aquecido em banho de silicone, a 50 e $60^{\circ} \mathrm{C}$, por 15 e 30 minutos, com agitação constante de 4500 rpm, centrifugado a $7500 \times g$ (Sorvall Instruments, RC5C, EUA) por 30 minutos, para separar o óleo, os sólidos e a água. Uma nova centrifugação foi realizada, nas mesmas condições, para remover traços de água. O óleo obtido foi mantido sob congelamento a $-80{ }^{\circ} \mathrm{C}$, até sua caracterização e purificação.

O processamento consistiu da passagem da matéria-prima à temperatura ambiente por três linhas horizontais de processamento, cada uma localizada no interior de um cozinhador, operando a $85-90{ }^{\circ} \mathrm{C}$ por 2 minutos. O material cozido foi separado em óleo, água e proteínas usando-se uma centrífuga operando a $85^{\circ} \mathrm{C}$. O óleo foi estabilizado adicionando-se $250 \mathrm{ppm}$ do antioxidante palmitato de ascorbila (PA) (TIMMHEINRICH et al., 2007), armazenado a $-80^{\circ} \mathrm{C}$ e reservado para análise. Foram utilizados também 12,5 L de óleo de vísceras e $6 \mathrm{~L}$ de óleo de fígado, ambos produzidos durante o outono. Também foram utilizados $10 \mathrm{~L}$ de óleo de vísceras, produzidos na primavera.

\subsubsection{Metodologia analítica}

\subsubsection{Caracterização do fígado}

Foram utilizados cerca de $500 \mathrm{~g}$ de fígado (22-26 unidades), sendo descongelados e homogeneizados para cada réplica. A composição proximal do fígado foi realizada em triplicata. $O$ conteúdo de umidade ( $\left.N^{\circ} .952,08\right)$ e cinzas $\left(N^{\circ} .938,08\right)$ foi determinado usando-se metodologia descrita pela AOAC (HORWITZ, 2005). O nitrogênio (\%N) foi determinado pelo analisador de nitrogênio Leco FP 2000 (LECO Co., St. Joseph, MO) e a proteína total, calculada pelo fator 6,25, segundo a AOAC (HORWITZ, 2005). Os lipídios totais foram calculados pelo método de Folch et al. (1957) e avaliados gravimetricamente.

Para a determinação do perfil de ácidos graxos, os ésteres metílicos de ácidos graxos (EMAG) foram preparados usando-se hidróxido de potássio e metanol, como descrito por Maxwell e Marmer (1983), com o éster metílico do ácido tricosanoico (C23:0) como padrão interno. Os EMAG foram mantidos em frascos âmbar de $1,5 \mathrm{~mL}$ e analisados em cromatógrafo a gás (CG) (Agilent Technologies, Wilmington, DE), provido de coluna capilar

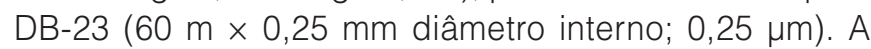
injeção no CG foi realizada automaticamente no volume de $1 \mu \mathrm{L}$. As condições cromatográficas seguiram conforme descrito por Bechtel e Oliveira (2006) e as análises foram feitas em triplicata. 


\subsubsection{Caracterização do óleo}

Os ácidos graxos livres (AGL) (Ca 5a-40), o índice de peróxidos (IP) (Cd 8b-90), o valor de anisidina (VA) (Cd 18-90), o valor de ácido tiobarbitúrico (TBA) (Cd 19-90) e o conteúdo de fósforo (CF) (Ca 12-55) foram analisados segundo a AOAC (FIRESTONE, 2004), em duplicata, da mesma forma que a atividade de água $\left(a_{w}\right)$, no medidor AquaLab, model Series 3TE (Decagon Devices, Inc., Washington, EUA) adaptado de Sathivel (2005); a cor foi medida em colorímetro Gardner Delta Comparator (BYK Gardner, Columbia, EUA) (BIMBO, 2009).

Triacilgliceróis (TAG), 1,2-diacilgliceróis (1,2-DAG), monoacilgliceróis (MAG), ácidos graxos livres (AGL), 1,3-diacilgliceróis (1,3-DAG), esteróis (ST) e fosfolipídios $(\mathrm{PL})$ foram quantificados usando-se $\mathrm{O}$ analisador latroscan TM TLC/FID modelo MK-6s (latron Laboratories Inc., Tókio, Japão), com base em Parrish (1987) e Oliveira e Bechtel (2005), em duplicata para cada amostra de óleo.

As vitaminas $A, D$ e $E$ foram determinadas usando os métodos NOVT 3.1 (Teste Analítico Kraft, USA), número 995.05 (HORWITZ, 2005) e o descrito por Kayden et al. (1983), respectivamente.

\subsubsection{Análise estatística}

Os dados obtidos foram analisados pela análise de variância e pelo teste de Tukey, no nível de 5 \% de significância, utilizando-se o programa Statistica for Windows versão 9.0 (StatSoft Inc., Tulsa, OK).

\section{Resultados e discussão}

\subsection{Composição do fígado de Alaska pollock}

A Tabela 1 apresenta a composição química do fígado de Alaska pollock em duas estações.

Tabela 1. Composição química ( $\mathrm{g} .100 \mathrm{~g}^{-1}$ ) de fígado de Alaska pollock capturado no outono e na primavera.

\begin{tabular}{lrr} 
Componentes $\mathbf{( g . 1 0 0 ~ \mathbf { ~ g } ^ { - 1 } )}$ & \multicolumn{1}{c}{ Outono } & Primavera \\
\hline Lipídios & $50,0 \pm 1,1^{\mathrm{a}}$ & $40,9 \pm 0,5^{\mathrm{b}}$ \\
Umidade & $39,9 \pm 0,5^{\mathrm{b}}$ & $48,2 \pm 0,7^{\mathrm{a}}$ \\
Proteína & $9,4 \pm 0.7^{\mathrm{b}}$ & $10,0 \pm 0,2^{\mathrm{a}}$ \\
Minerais & $0,7 \pm 0,1^{\mathrm{a}}$ & $0,9 \pm 0,07^{\mathrm{a}}$ \\
\hline
\end{tabular}

Letras diferentes nas linhas indicam que os resultados são estatisticamente diferentes $(P<0,05)$.
O conteúdo de lipídios das amostras obtidas no outono foi $10 \%$ maior do que na primavera e o conteúdo de umidade foi inversamente proporcional ao conteúdo lipídico. Este resultado concorda com as perspectivas, pois o pollock acumula lipídios no tecido do fígado, não os acumulando no músculo como fonte de energia. De acordo com Oliveira e Bechtel (2005), o conteúdo lipídico do filé de pollock é de 0,8 $\pm 0,2 \%$. Esses autores reportaram $55 \%$ de lipídios presentes no fígado, mas não definiram a temporada de pesca na qual a amostragem foi conduzida. Os lipídios presentes nos fígados de pollock coletados no mês de novembro, determinados por Bechtel e Oliveira (2006), foram da ordem de 50,3 \%, enquanto que, num estudo mais detalhado, esses autores determinaram que foi de 52 e 55 \% nos fígados de fêmeas e machos, respectivamente (OLIVEIRA e BECHTEL, 2006). Dados presentes na literatura demonstram que 0 pollock acumula lipídios no fígado durante o verão e o outono, se preparando para o ciclo de reprodução, que ocorre no início da primavera (BECHTEL e OLIVEIRA, 2006). Aidos et al. (2002) e Ribeiro (2010) enfatizam que o teor de lipídios pode variar segundo o período de pesca, a região de captura, a estação do ano, a maturidade sexual e a saúde do animal.

A Tabela 2 apresenta variações nos ácidos graxos mais abundantes, que compõem aproximadamente 70 \% da fração lipídica do fígado de pollock de outono e primavera. O perfil completo dos ácidos graxos de pollock é descrito por Ribeiro (2010), segundo o qual os ácidos graxos de $\omega-3$ foram baixos, quando comparados aos lipídios presentes no fígado de bacalhau do Atlântico. De acordo com Oliveira e Bechtel (2006), a quantidade de AEP e ADH no fígado de pollock é de $157 \mathrm{mg}^{-\mathrm{g}^{-1}}$ de lipídios, quando foi observado um decréscimo de $10 \%$ no conteúdo de AEP e ADH entre os óleos de outono e primavera, indicando que fatores biológicos (estado de reprodução e tamanho) ou fatores ambientais (temperatura da água, estação do ano) influenciam a composição dos ácidos graxos dos lipídios extraídos de tecidos de origem marinha. Durante o verão, o pollock se alimenta de quantidades significativas de krill, que contém alto teor de ácidos eicosanoico e docosanoico monoinsaturados.

Tabela 2. Perfil dos ácidos graxos da fase lipídica do fígado de Alaska pollock expresso em mg.g-1 de óleo e porcentagem peso/peso.

\begin{tabular}{|c|c|c|c|c|}
\hline \multirow[b]{2}{*}{ Ácidos graxos } & \multicolumn{4}{|c|}{ Estações } \\
\hline & $\begin{array}{l}\text { Outono } \\
\mathrm{mg} \mathrm{g}^{-1}\end{array}$ & $\begin{array}{c}\text { Outono } \\
\% \text { peso/peso }\end{array}$ & $\begin{array}{c}\text { Primavera } \\
\mathrm{mg} \mathrm{g}^{-1}\end{array}$ & $\begin{array}{l}\text { Primavera } \\
\% \text { peso/peso }\end{array}$ \\
\hline $20: 5 \omega-3$ (AEP) & $71,2 \pm 1,2^{\mathrm{a}}$ & $9,7 \pm 0,1^{a}$ & $59,4 \pm 1,2^{b}$ & $9,4 \pm 0,2^{b}$ \\
\hline $20: 6 \omega-3(A D H)$ & $35,2 \pm 0,7^{a}$ & $4,8 \pm 0,1^{a}$ & $30,6 \pm 0,6^{b}$ & $4,8 \pm 0,1^{a}$ \\
\hline$\Sigma$ AGPI & $140,6 \pm 2,3^{a}$ & $19,1 \pm 0,1^{a}$ & $117,9 \pm 2,2^{b}$ & $18,8 \pm 0,3^{b}$ \\
\hline$\Sigma \omega-3$ & $117,8 \pm 2,0^{a}$ & $16,0 \pm 0,1^{a}$ & $99,1 \pm 1,9^{b}$ & $15,8 \pm 0,2^{b}$ \\
\hline$\Sigma \omega-6$ & $14,9 \pm 0,1^{a}$ & $2,0 \pm 0,0^{a}$ & $10,3 \pm 0,2^{b}$ & $1,6 \pm 0,0^{b}$ \\
\hline$\omega-3 / \omega-6$ & $7,9 \pm 0,1^{b}$ & $7,9 \pm 0,1^{b}$ & $9,6 \pm 0,0^{a}$ & $9,6 \pm 0,0^{a}$ \\
\hline
\end{tabular}

AGPI: ácidos graxos poli-insaturados; AEP: ácido eicosapentaenoico; ADH: ácido docosahexaenoico. Letras diferentes entre as linhas de mesma unidade de peso indicam diferença estatística $(P<0,05)$. 
Avaliação do processamento a baixas temperaturas do óleo de fígado de Alaska pollock (Theragra chalcogramma) RIBEIRO, V. A. et al.

\subsection{Caracterização do óleo de pescado}

Os parâmetros do processo de extração de óleo do fígado em diferentes temperaturas, tempos e estações do ano são mostrados na Tabela 3.

Pôde-se observar que o óleo de outono apresentou um rendimento superior em lipídios se comparado com o óleo da primavera. O maior rendimento encontrado foi $52 \%$, quando foi processado a $60{ }^{\circ} \mathrm{C}$ por 30 minutos; portanto, esta foi a única condição usada para extrair os óleos da primavera. De acordo com Chantachum et al. (2000), baixas temperaturas podem influenciar na obtenção de rendimento menor, pois as células lipídicas no pescado são rompidas em altas temperaturas de processamento $\left(85^{\circ} \mathrm{C}\right)$. Pode ocorrer a desnaturação irreversível das proteínas submetidas a altas temperaturas (90 a $100^{\circ} \mathrm{C}$ ) por um período prolongado. O experimento mostrou que o tempo prolongado influenciou mais do que as baixas temperaturas no processo de extração, pois o maior rendimento foi obtido quando as condições incluíam tempos prolongados.

Conforme a Tabela 4, o óleo de fígado produzido em laboratório, em diferentes condições, não apresentou diferença estatística na porcentagem de $A G L$, indicando que o tempo, a temperatura e a variação da estação pouco influenciaram na atividade enzimática. Porém, verificou-se que o valor de AGL obtido foi maior se comparado ao óleo de fígado e similar ao óleo de vísceras, ambos produzidos pelo navio processador. De acordo com Bimbo (2009), o percentual máximo de AGL aceitável para óleo de pescado bruto é $5 \%$; portanto, todos os óleos encontram-se abaixo do valor máximo de AGL.

Os valores de IP, VA e TBA (Tabela 4) mostraram que o óleo de laboratório apresentou diferença quando submetido a diferentes tratamentos, indicando que o tempo, a temperatura e a estação do ano influenciaram na possível oxidação lipídica primária e secundária. Em

Tabela 3. Parâmetros do processo de extração do óleo de fígado de Alaska pollock em diferentes temperaturas, tempos e duas estações do ano.

\begin{tabular}{|c|c|c|c|c|c|}
\hline \multirow{3}{*}{ Parâmetros } & \multicolumn{5}{|c|}{ Amostra } \\
\hline & D1 & D2 & D3 & D4 & E \\
\hline & $50{ }^{\circ} \mathrm{C} / 15 \mathrm{~min}$ & $50^{\circ} \mathrm{C} / 30 \mathrm{~min}$ & $60^{\circ} \mathrm{C} / 15 \mathrm{~min}$ & $60^{\circ} \mathrm{C} / 30 \mathrm{~min}$ & $60^{\circ} \mathrm{C} / 30 \mathrm{~min}$ \\
\hline Peso da amostra (g) & $503,5 \pm 1,3$ & $504,5 \pm 5,4$ & $502,0 \pm 2,1$ & $502,9 \pm 1,8$ & $506,1 \pm 3,1$ \\
\hline Peso I de óleo (1) & $256,0 \pm 2,3$ & $293,4 \pm 10,2$ & $259,7 \pm 2,1$ & $289,1 \pm 2,4$ & $250,4 \pm 1,0$ \\
\hline Peso II de óleo (2) & $225,2 \pm 0,9$ & $257,8 \pm 6,0$ & $230,8 \pm 0,6$ & $263,1 \pm 4,1$ & $213,0 \pm 3,3$ \\
\hline $\begin{array}{l}\text { Peso da fração } \\
\text { proteica }\end{array}$ & $106,7 \pm 3,1$ & $82,6 \pm 1,3$ & $79,4 \pm 2,1$ & $82,0 \pm 0,8$ & $121,7 \pm 2,0$ \\
\hline Rendimento I (3) & $21,1 \pm 0,6^{a}$ & $16,4 \pm 0,3^{b}$ & $15,7 \pm 0,4^{b}$ & $16,3 \pm 0,2^{b}$ & $23,3 \pm 0,4^{*}$ \\
\hline Rendimento II (4) & $44,6 \pm 0,3^{d}$ & $51,1 \pm 0,9^{b}$ & $46,0 \pm 0,2^{c}$ & $52,3 \pm 0,8^{a^{*}}$ & $41,3 \pm 0,9$ \\
\hline
\end{tabular}

D1: óleo de outono a $50{ }^{\circ} \mathrm{C} / 15 \mathrm{~min}$; D2: óleo de outono a $50{ }^{\circ} \mathrm{C} / 30 \mathrm{~min}$; D3: óleo de outono a $60{ }^{\circ} \mathrm{C} / 15 \mathrm{~min}$; D4: óleo de outono a $60{ }^{\circ} \mathrm{C} / 30 \mathrm{~min}$; E: óleo de primavera; (1)Peso I de óleo: peso depois da primeira centrifugação; (2)Peso II de óleo: peso depois da segunda centrifugação; (3) Rendimento I: Rendimento da fração proteica; (4)Rendimento II: Rendimento do óleo; *Indica diferença estatística entre os óleos D4 e E; letras diferentes entre as linhas indicam diferença estatística $(P<0,05)$.

Tabela 4. Caracterização dos óleos de fígado e vísceras processados no navio American Triumph, e óleos de fígado de Alaska pollock produzidos em laboratório em diferentes temperaturas $\left(50\right.$ e $60^{\circ} \mathrm{C}$ ) e tempo (15 a 30 minutos), provenientes das estações outono e primavera.

\begin{tabular}{|c|c|c|c|c|c|c|c|}
\hline \multirow[b]{2}{*}{ Amostra } & \multirow[b]{2}{*}{ Tratamento } & \multicolumn{6}{|c|}{ Índice } \\
\hline & & $\begin{array}{c}\text { AGL } \\
\text { (\% ácido oleico) }\end{array}$ & $\begin{array}{c}\text { IP } \\
\text { (meq/kg) }\end{array}$ & VA & $\begin{array}{c}\text { TBA } \\
\text { (mg MDA/kg) }\end{array}$ & $a_{w}$ & $\begin{array}{c}\text { CF } \\
(\mathrm{ppm})\end{array}$ \\
\hline D1 & $50^{\circ} \mathrm{C} / 15 \mathrm{~min}$ & $0,4 \pm 0,1^{a}$ & $12,9 \pm 0,9^{b}$ & $22,0 \pm 1,7^{c}$ & $0,6 \pm 0,1^{a}$ & $0,6 \pm 0,0^{a}$ & $14,8 \pm 1,1^{a}$ \\
\hline D2 & $50^{\circ} \mathrm{C} / 30 \mathrm{~min}$ & $0,4 \pm 0,2^{a}$ & $13,3 \pm 1,2^{\mathrm{ab}}$ & $22,6 \pm 2,5^{c}$ & $0,6 \pm 0,1^{\mathrm{a}}$ & $0,5 \pm 0,0^{b}$ & $11,3 \pm 0,9^{b}$ \\
\hline D3 & $60^{\circ} \mathrm{C} / 15 \mathrm{~min}$ & $0,4 \pm 0,2^{a}$ & $14,2 \pm 0,5^{a b}$ & $25,2 \pm 2,1^{b}$ & $0,7 \pm 0,1^{a}$ & $0,5 \pm 0,0^{b}$ & $11,3 \pm 1,8^{b}$ \\
\hline D4 & $60^{\circ} \mathrm{C} / 30 \mathrm{~min}$ & $0,4 \pm 0,1^{a}$ & $14,3 \pm 1,5^{a^{*}}$ & $28,0 \pm 2,1^{a^{*}}$ & $0,7 \pm 0,1^{a^{*}}$ & $0,4 \pm 0,0^{c}$ & $9,9 \pm 1,4^{c}$ \\
\hline$E$ & $60^{\circ} \mathrm{C} / 30 \mathrm{~min}$ & $0,4 \pm 0,2$ & $13,5 \pm 0,7$ & $26,1 \pm 1,5$ & $0,5 \pm 0,0$ & $0,7 \pm 0,0^{*}$ & $12,2 \pm 1,0^{*}$ \\
\hline A & $90{ }^{\circ} \mathrm{C} / 2 \mathrm{~min}$ & $0,4 \pm 0,0$ & $6,3 \pm 0,6$ & $22,0 \pm 0,2$ & $0,4 \pm 0,0$ & $0,7 \pm 0,0$ & $7,8 \pm 0,4$ \\
\hline B & $90^{\circ} \mathrm{C} / 2 \mathrm{~min}$ & $0,2 \pm 0,1$ & $4,7 \pm 0,0$ & $21,0 \pm 0,4$ & $0,2 \pm 0,0$ & $0,5 \pm 0,0$ & $8,1 \pm 0,7$ \\
\hline C & $90^{\circ} \mathrm{C} / 2 \mathrm{~min}$ & $0,3 \pm 0,4$ & $5,7 \pm 0,2$ & $23,8 \pm 0,2$ & $0,2 \pm 0,0$ & $0,7 \pm 0,0$ & $7,7 \pm 1,0$ \\
\hline
\end{tabular}

AGL: ácidos graxos livres; IP: índice de peróxidos; VA: valor de anisidina; TBA: valor de 2-ácido tiobarbitúrico (mg de malonaldeído por kg de amostra); $a_{w}$ : atividade de água; CF: conteúdo de fósforo; A: óleo de vísceras produzido no outono; B: óleo de fígado produzido no outono; C: óleo de vísceras produzido na primavera; D1,2,3,4: óleo de fígado produzido em laboratório durante o outono; E: óleo de fígado produzido em laboratório durante a primavera; *Indica diferença estatística entre os óleos D4 e E. Diferentes letras entre as colunas indicam diferença estatística $(P<0,05)$. 
Avaliação do processamento a baixas temperaturas do óleo de fígado de Alaska pollock (Theragra chalcogramma) RIBEIRO, V. A. et al.

geral, os índices do óleo de fígado de laboratório foram maiores se comparados com o óleo de fígado e o óleo de vísceras processados no navio, o que indica que o tempo prolongado de aquecimento durante o processamento em laboratório pode ter favorecido as reações de oxidação. De acordo com Boran et al. (2006) e Bimbo (2009), os valores máximos aceitáveis de IP, VA e TBA para óleo de pescado bruto são 20 meg. $\mathrm{kg}^{-1}$, 20 e 7-8 mg MDA. $\mathrm{kg}^{-1}$, respectivamente. Todos os óleos apresentaram valores abaixo desses limites.

Os valores de $\mathrm{a}_{\mathrm{w}}$ dos óleos extraídos em laboratório foram menores quando comparado com o óleo processado no navio (Tabela 4), sendo observado um decréscimo na atividade de água com o aumento da temperatura e do tempo de extração. De acordo com Sathivel (2005), a a do óleo extraído da cabeça de salmão vermelho e rosa foi 0,57 e 0,53, respectivamente. Verificou-se que o óleo de fígado produzido em laboratório apresentou valores similares, exceto o óleo extraído à baixa temperatura. O conteúdo de fósforo no óleo de fígado produzido em laboratório foi superior ao óleo processado no navio. De acordo com Bimbo (2009), a taxa de concentração de fósforo aceitável para óleo bruto varia de 5 a 100 ppm; portanto, todos os óleos mostraram valores dentro do padrão.

Pela Tabela 5, verificou-se que o óleo é composto na maior parte de triacilgliceróis e que o conteúdo de diacilglicerol e esterol foi inferior no óleo processado no navio, em relação ao óleo produzido em laboratório. Além disso, a perda de triacilgliceróis do óleo de fígado produzido em laboratório aumentou com a elevação da temperatura, indicando a forte influência dessa variável na concentração de triacilgliceróis. Segundo Boran et al. (2006), triacilgliceróis, tri-ésteres de ácidos graxos e gliceróis são os principais constituintes do óleo. O restante consiste parcialmente de glicídios, como mono e diacilgliceróis, ácidos graxos livres ( $A G L)$ e outros compostos menores. Assim, os óleos A, B, C, D4 e E apresentaram essas características.

Observou-se, na Tabela 6, que os resultados para ácidos graxos AEP e ADH foram mais elevados no óleo de fígado extraído a $60^{\circ} \mathrm{C}$ do que a $50^{\circ} \mathrm{C}$. Foi observado

Tabela 5. Conteúdo de lipídios (\%) por classes do óleo de fígado e vísceras processado no navio American Triumph e dos óleos de fígado produzidos em laboratório, em diferentes temperaturas (50 e $60^{\circ} \mathrm{C}$ ) e tempo (15 a 30 minutos) de extração.

\begin{tabular}{|c|c|c|c|c|c|c|}
\hline \multirow{2}{*}{ Amostra } & \multirow{2}{*}{ Tratamento } & \multicolumn{5}{|c|}{ Conteúdo de lipídios (\%) } \\
\hline & & TAG & AGL & DAG/ST & MAG & PL \\
\hline D1 & $50^{\circ} \mathrm{C} / 15 \mathrm{~min}$ & $86,8 \pm 1,7^{c}$ & $5,4 \pm 1,7^{a}$ & $4,7 \pm 0,8^{a}$ & $0,5 \pm 0,3^{a}$ & $2,4 \pm 1,1^{a}$ \\
\hline D2 & $50^{\circ} \mathrm{C} / 30 \mathrm{~min}$ & $89,3 \pm 0,5^{b}$ & $4,9 \pm 0,5^{b}$ & $3,7 \pm 0,4^{b}$ & $0,3 \pm 0,0^{a}$ & $1,6 \pm 0,3^{b}$ \\
\hline D3 & $60^{\circ} \mathrm{C} / 15 \mathrm{~min}$ & $88,6 \pm 0,7^{b}$ & $5,5 \pm 0,7^{a}$ & $3,5 \pm 0,2^{b}$ & $0,4 \pm 0,3^{a}$ & $1,7 \pm 0,8^{b}$ \\
\hline D4 & $60^{\circ} \mathrm{C} / 30 \mathrm{~min}$ & $91,5 \pm 0,9^{a}$ & $3,5 \pm 1,2^{c}$ & $3,4 \pm 0,6^{b *}$ & $0,5 \pm 0,8^{a}$ & $0,9 \pm 0,4^{c}$ \\
\hline$E$ & $60^{\circ} \mathrm{C} / 30 \mathrm{~min}$ & $93,0 \pm 1,4^{*}$ & $4,4 \pm 0,7^{*}$ & $1,6 \pm 1,2$ & $<0,01$ & $0,9 \pm 0,4$ \\
\hline A & $90{ }^{\circ} \mathrm{C} / 2 \mathrm{~min}$ & $99,4 \pm 0,1$ & $<0,01$ & $0,5 \pm 0,0$ & $<0,01$ & $<0,01$ \\
\hline B & $90{ }^{\circ} \mathrm{C} / 2 \mathrm{~min}$ & $100,0 \pm 0,0$ & $<0,01$ & $<0,01$ & $<0,01$ & $<0,01$ \\
\hline C & $90^{\circ} \mathrm{C} / 2 \min$ & $100,0 \pm 0,0$ & $<0,01$ & $<0,01$ & $<0,01$ & $<0,01$ \\
\hline
\end{tabular}

TAG: triacilglicerol; AGL: ácidos graxos livres; DAG/ST: diacilglicerol e esterol; MAG: monoacilglicerol; PL: fosfolipídios; A: óleo de vísceras produzido no outono; B: óleo de fígado produzido durante o outono; C: óleo de vísceras produzido na primavera; D1,2,3,4: óleo de fígado produzido em laboratório durante o outono; E: óleo de fígado produzido em laboratório durante a primavera. *Indica diferença estatística entre os óleos D4 e E. Diferentes letras entre as colunas indicam diferença estatística $(P<0,05)$.

Tabela 6. Perfil de ácidos graxos do óleo de fígado e vísceras processado no navio American Triumph e óleos de fígado produzidos em laboratório (\% peso/peso).

\begin{tabular}{|c|c|c|c|c|c|c|c|}
\hline \multirow[b]{2}{*}{ Amostra } & \multirow[b]{2}{*}{ Tratamento } & \multicolumn{6}{|c|}{ Ácidos graxos } \\
\hline & & $\begin{array}{c}20: 5 \omega-3 \\
\text { (AEP) }\end{array}$ & $\begin{array}{c}20: 6 \omega-3 \\
\text { (ADH) }\end{array}$ & $\Sigma$ AGPI & $\Sigma \omega-3$ & $\Sigma \omega-6$ & $\omega-3 / \omega-6$ \\
\hline D1 & $50^{\circ} \mathrm{C} / 15 \mathrm{~min}$ & $8,9 \pm 0,5^{c}$ & $4,4 \pm 0,34^{b}$ & $18,6 \pm 1,5^{c}$ & $13,5 \pm 0,1^{c}$ & $1,3 \pm 0,1^{b}$ & $10,0 \pm 0,2^{b}$ \\
\hline D2 & $50^{\circ} \mathrm{C} / 30 \mathrm{~min}$ & $9,8 \pm 0,1^{a}$ & $4,7 \pm 0,0^{a}$ & $19,1 \pm 0,2^{b}$ & $13,5 \pm 0,2^{c}$ & $1,1 \pm 0,3^{c}$ & $12,1 \pm 0,1^{a}$ \\
\hline D3 & $60^{\circ} \mathrm{C} / 15 \mathrm{~min}$ & $9,3 \pm 0,2^{b}$ & $4,5 \pm 0,1^{b}$ & $18,4 \pm 0,4^{c}$ & $15,9 \pm 0,1^{b}$ & $1,5 \pm 0,0^{a}$ & $10,5 \pm 0,3^{b}$ \\
\hline D4 & $60^{\circ} \mathrm{C} / 30 \mathrm{~min}$ & $9,6 \pm 0,1^{a b}$ & $4,8 \pm 0,14^{\mathrm{a}}$ & $19,3 \pm 0,2^{a}$ & $16,6 \pm 1,8^{a}$ & $1,3 \pm 0,1^{b}$ & $12,3 \pm 0,1^{a^{\star}}$ \\
\hline$E$ & $60^{\circ} \mathrm{C} / 30 \mathrm{~min}$ & $11,3 \pm 0,4^{*}$ & $5,1 \pm 0,17^{\star}$ & $22,0 \pm 0,4^{*}$ & $18,4 \pm 0,3^{*}$ & $2,1 \pm 0,0^{*}$ & $8,7 \pm 0,1$ \\
\hline A & $90^{\circ} \mathrm{C} / 2 \mathrm{~min}$ & $10,9 \pm 0,1$ & $4,9 \pm 0,0$ & $19,9 \pm 0,1$ & $18,3 \pm 0,1$ & $1,5 \pm 0,2$ & $11,7 \pm 1,9$ \\
\hline B & $90^{\circ} \mathrm{C} / 2 \mathrm{~min}$ & $10,8 \pm 0,0$ & $4,8 \pm 0,0$ & $19,7 \pm 0,3$ & $18,2 \pm 0,1$ & $1,5 \pm 0,2$ & $11,9 \pm 1,4$ \\
\hline C & $90^{\circ} \mathrm{C} / 2 \mathrm{~min}$ & $10,0 \pm 0,0$ & $5,1 \pm 0,0$ & $20,9 \pm 0,1$ & $17,2 \pm 0,0$ & $2,1 \pm 0,0$ & $9,4 \pm 0,2$ \\
\hline
\end{tabular}

AGPI: ácidos graxos poliinsaturados; AEP: ácidos eicosapentaenoico; ADH: ácidos docosahexaenoico; A: óleo de vísceras produzido no outono; B: óleo de fígado produzido durante o outono; C: óleo de vísceras produzido na primavera; D: óleo de fígado produzido em laboratório durante o outono; E: óleo de fígado produzido em laboratório durante a primavera; *Indica diferença estatística entre os óleos D4 e E; Diferentes letras entre as linhas indicam diferença estatística $(P<0,05)$ 
que o óleo processado no navio apresentou concentração maior em relação ao óleo produzido em laboratório, indicando que a temperatura e o tempo influenciaram no processo. O óleo de fígado e vísceras produzido durante o outono apresentou percentual de AEP e de ADH de $15,5 \%$, enquanto que, em óleos da primavera, o valor de AEP e ADH decresceu para 9,5\%.

Os óleos de outono apresentaram um percentual de AEP e ADH próximo ao citado por Hajaltason e Haraldsson (2006) e Bimbo (2007), pois, segundo esses autores, foi encontrada uma porcentagem de AEP e ADH de $19 \%$ no fígado de bacalhau e de $16 \%$ no óleo de fígado de pollock. As variações na porcentagem de ácidos graxos podem ocorrer em amostras de diferentes localizações. Desta forma, fatores biológicos (sexo, estádio ou fase de reprodução e tamanho) ou fatores ambientais (temperatura da água e estação do ano) podem influenciar a composição dos ácidos graxos (BIMBO, 2007). O percentual de AGPI foi maior em óleo processado no navio do que o de laboratório, porém a porcentagem de ácidos graxos monoinsaturados (AGMI) comportou-se inversamente aos AGPI. Os resultados

Tabela 7. Cor apresentada pelos diferentes óleos de Alaska pollock.

\begin{tabular}{clc} 
Amostra & Tratamento & Valor GDC \\
\hline D1 & $50^{\circ} \mathrm{C} / 15 \mathrm{~min}$ & 9 \\
D2 & $50^{\circ} \mathrm{C} / 3 \mathrm{~min}$ & 5 \\
D3 & $60^{\circ} \mathrm{C} / 15 \mathrm{~min}$ & 7 \\
D4 & $60^{\circ} \mathrm{C} / 30 \mathrm{~min}$ & 4 \\
$\mathrm{E}$ & $60^{\circ} \mathrm{C} / 30 \mathrm{~min}$ & 4 \\
$\mathrm{~A}$ & $90^{\circ} \mathrm{C} / 2 \mathrm{~min}$ & 12 \\
$\mathrm{~B}$ & $90^{\circ} \mathrm{C} / 2 \mathrm{~min}$ & 4 \\
$\mathrm{C}$ & $90^{\circ} \mathrm{C} / 2 \mathrm{~min}$ & 7 \\
\hline
\end{tabular}

GDC: Valor do Gardner Delta Comparator; A: óleo de vísceras produzido no outono; B: óleo de fígado produzido durante o outono; C: óleo de vísceras produzido na primavera; D: óleo de fígado produzido em laboratório durante o outono; E: óleo de fígado produzido em laboratório na primavera. estão de acordo com estudos prévios (BECHTEL e OLIVEIRA, 2006; OLIVEIRA e BECHTEL, 2006).

Por meio da Tabela 7, foi verificado que os óleos de fígado se mostraram mais claros que os de vísceras, estes mais avermelhados, sendo ambos produzidos durante o outono no navio. Pôde-se observar que a coloração foi mais amarelada do que a dos óleos de vísceras produzidos na primavera. O óleo de fígado produzido a 50 e $60{ }^{\circ} \mathrm{C}$ por 30 minutos foi mais claro que os óleos produzidos a 15 minutos. Porém, não foi encontrada diferença na coloração em óleos produzidos em laboratório entre ambas as estações. Acredita-se que o tempo poderia ter influenciado mais do que a baixa temperatura na remoção dos pigmentos, pois a coloração amarela foi mais intensa nos óleos obtidos em tempo prolongado. Todos os óleos apresentaram uma coloração inferior ao limite de 14, recomendado pelo método de Gardner (BIMBO, 2007). A variação na cor do óleo produzido no outono e na primavera está relacionada com a alimentação rica em krill (WILSON et al., 2009), excelente fonte de pigmentos naturais e vitaminas (BIMBO, 2007). Na medida em que o outono é o período em que a larva do krill atinge a maturidade, há disponibilidade de alimento para o pollock ingerir elevada quantidade de pigmentos.

A Tabela 8 mostra os níveis de vitaminas em diferentes tratamentos. Foi visualizada a tendência de o conteúdo de vitamina A ser mais alto no óleo de fígado do que no óleo de vísceras. Os subprodutos de pescado contêm elevados níveis de retinol, pois o fígado é reservatório de vitamina $A$. Os teores de vitamina $A$ determinados em todos os óleos analisados superaram a referência de 10 a $50 \mu \mathrm{g}$ de retinol. $\mathrm{g}^{-1}$ óleo de fígado. O elevado tempo de estocagem da matéria-prima poderia ter formado precursores de vitamina $A$, depois convertidos em retinol (WU; BECHTEL, 2009).

Foram encontrados valores baixos de vitamina E nos óleos de fígado extraídos em laboratório, provavelmente em razão da degradação dessa vitamina durante o processo de extração por tempo prolongado (15 e 30 minutos) e sem vácuo ou atmosfera inerte. Quando

Tabela 8. Conteúdo de vitaminas lipossolúveis do óleo processado no navio American Triumph e óleos produzidos em escala laboratorial.

\begin{tabular}{|c|c|c|c|c|c|c|c|}
\hline \multirow{2}{*}{ Amostra } & \multirow{2}{*}{ Tratamento } & \multicolumn{3}{|c|}{ Vitaminas ( $\mu \mathrm{g} \cdot \mathrm{g}^{-1}$ de óleo) } & \multicolumn{3}{|c|}{ Vitaminas (UI) } \\
\hline & & A & D & E & A & D & E \\
\hline A & $90^{\circ} \mathrm{C} / 2 \mathrm{~min}$ & 742,0 & 9,0 & 39,6 & 2473,3 & 360 & 0,059 \\
\hline$A^{*}$ & $90{ }^{\circ} \mathrm{C} / 2 \min$ & 720,2 & 8,7 & 17,8 & 2400,7 & 348 & 0,027 \\
\hline B & $90{ }^{\circ} \mathrm{C} / 2 \min$ & 1272,5 & 6,9 & 49,6 & 3979,7 & 276 & 0,074 \\
\hline$B^{*}$ & $90^{\circ} \mathrm{C} / 2 \mathrm{~min}$ & 1193,9 & 6,3 & 29,7 & 4241,7 & 252 & 0,044 \\
\hline D1 & $50^{\circ} \mathrm{C} / 15 \mathrm{~min}$ & 702,0 & 5,7 & 6,3 & 2340,0 & 228 & 0,009 \\
\hline D2 & $50^{\circ} \mathrm{C} / 30 \mathrm{~min}$ & 853,9 & 8,1 & 8,7 & 2846,3 & 324 & 0,013 \\
\hline D3 & $60^{\circ} \mathrm{C} / 15 \mathrm{~min}$ & 593,1 & 6,3 & 12,3 & 1977,0 & 252 & 0,018 \\
\hline D4 & $60^{\circ} \mathrm{C} / 30 \mathrm{~min}$ & 565,0 & 4,8 & 8,2 & 1883,3 & 192 & 0,012 \\
\hline
\end{tabular}

A: óleo de vísceras produzido no outono; B: óleo de fígado produzido durante outono; D1,2,3,4: óleo de fígado produzido em laboratório durante o outono; UI: unidade internacional usada para quantificar as vitaminas. ${ }^{*}$ ndica que o óleo testado não foi tratado com antioxidante (palmitato de ascorbila). 
Avaliação do processamento a baixas temperaturas do óleo de fígado de Alaska pollock (Theragra chalcogramma) RIBEIRO, V. A. et al.

comparados com os óleos produzidos no navio ( $A$ e $B$ com e sem antioxidante), em que o tempo do processo foi 2 minutos, foram encontrados conteúdos significativos de vitamina $E$. O conteúdo de vitamina $E$ do óleo $A$ foi aproximadamente o dobro do valor encontrado para o óleo $A^{*}$. Da mesma maneira, o óleo B apresentou valor superior para esta vitamina em comparação ao óleo $\mathrm{B}^{*}$, sugerindo a eficácia do antioxidante escolhido na preservação da vitamina; porém, note-se que todos os valores obtidos foram inferiores aos encontrados por Wu e Bechtel (2009), de $172 \mu \mathrm{g} . \mathrm{g}^{-1}$ óleo.

\section{Conclusões}

Foi verificada uma variação na composição química e no perfil de ácidos graxos dos fígados de Alaska pollock capturados e processados em diferentes estações do ano. O maior rendimento do processo de extração encontrado foi $52 \%$, quando utilizado $60^{\circ} \mathrm{C}$ por 30 minutos, e o menor rendimento encontrado foi $44 \%$, a $50{ }^{\circ} \mathrm{C}$ por 15 minutos. Os índices de qualidade do óleo produzido em laboratório apresentaram-se dentro dos padrões aceitáveis para o consumo humano, mas foram inferiores aos óleos produzidos no navio processador, o que indica que o tempo de estocagem, o congelamento e o descongelamento da amostra, e o tempo de extração tiveram um efeito negativo sobre a qualidade oxidativa do óleo. O teor de ácidos graxos poli-insaturados presente nos óleos produzidos em laboratório e no navio processador situou-se ao redor de $80 \%$. A estação do ano influenciou na coloração dos óleos, ficando mais avermelhados no outono e mais amarelados na primavera.

\section{Agradecimentos}

Os autores agradecem ao Pollock Conservation Cooperative Research Center, University of Alaska Fairbanks, Estados Unidos, pelo apoio financeiro e pela Bolsa de Estudos concedidos.

\section{Referências}

AIDOS, I.; PADT, A. V.; LUTTEN, J. B.; BOOM, R. M. Seasonal changes in crude and lipid composition of herring fillets, byproducts, and respective produced oils. Journal of Agricultural and Food Chemistry, Davis, v. 50, n. 16, p. 45894599, 2002.

BECHTEL, P. J.; OLIVEIRA, A. C. M. Chemical characterization of livers from fish species harvested in Alaska. Journal of Food Science, Chicago, v. 71, n. 6, p. S480-486, 2006. http://dx.doi. org/10.1111/j.1750-3841.2006.00076.x

BECHTEL, P. J. By-products from seafood processing for aquaculture and animal feeds. In: SHAHIDI, F. (Ed.). Maximizing the Value of Marine By-Products. St. Johns: CRC Press, 2007. p. 435-445. http://dx.doi.org/10.1533/9781845692087.3.435
BIMBO, A. P. Processing of marine oils. In: BREIVIK, H. (Ed.). Long-Chain Omega-3 Specialty Oils. Bridgewater: The Oil Press, 2007. p. 86-95. http://dx. doi.org/10.1533/9780857097897

BIMBO, A. P. Alaska Seafood Byproducts: Potential Products, Markets and Competing Products. Anchorage: Alaska Fisheries Development Foundation, Inc., 2009. Disponível em: <http://www.afdf.org/pastresearch/2008byproductmktstudy. pdf>. Acesso em: 09 dez. 2011.

BORAN, G.; KARAC, H.; BORAN, M. Changes in the quality of fish oils due to storage temperature and time. Food Chemistry, Barking, v. 98, n. 4, p. 693-698, 2006.

CHANTACHUM, S.; BENJAKUL, S.; SRIWIRAT, N. Separation and quality of fish oil from precooked and non-precooked tuna heads. Food Chemistry, Barking, v. 69, n. 3, p. 289-294, 2000.

FERDOSH, S.; SARKER, Z. I.; SELAMAT, J.; YAZID, A. M.; ALFI, K.; NORULAINI, N. A. N. Fatty acid composition of fish oil extracted from different parts of Indian mackerel (Rastrelliger kanagurta) using various techniques of supercritical $\mathrm{CO}_{2}$ extraction. Food Chemistry, Barking, v. 120, n. 3, p. 879-885, 2010.

FIRESTONE, D. (Ed.). Official Methods of Analysis of the Association of Official Analytical Chemists. 5th ed. Champaign: AOAC, 2004

FOLCH, J.; LEES, M.; SLOANE-STANLEY, G. H. A simple method for isolation and purification of total lipids from animal tissues. Journal of Biological Chemistry, Rockville, v. 226, p. 497-509, 1957.

HAJALTASON, B.; HARALDSSON, G. G. Fish oil and lipids from marine source. In: GUNSTONE, F. D. (Ed.). Modifying lipids for use in food. Cambridge: Woodhead Publishing, 2006. p. 56-79.

HORWITZ, W. (Ed.). Official Methods of Analysis of the Association of Official Analytical Chemists. 16th ed. Washington: AOAC, 2005.

JACHAMANIÁN, I.; MARGENAT, L.; TORRES, A. I.; GROMPONE, M. A. Selectivity of supercritical $\mathrm{CO}_{2}$ in the fractionation of hake liver oil ethyl esters. Journal of the American Oil Chemists' Society, Urbana, v. 84, n. 6, p. 597-601, 2007

KAYDEN, H. J.; HATAM, L. J.; TRABEL, M. G. The measurement of nanograms of tocopherol from needle aspiration biopsies of adipose tissue. Journal of Lipid Research, Rockville, v. 24, p. 652-656, 1983.

MAXWELL, R. J.; MARMER, W. N. Systematic protocol for the accumulation of fatty acid data from multiple tissues sample: Tissue handling, lipid extraction, lipid class separation, and gas chromatographic analysis. Lipids, Urbana, v. 18, n. 7 , p. 453-459, 1983. http://dx.doi.org/10.1007/BF02535785

MARINE CONSERVATION SOCIETY - MCS. Fish On Line: Alaska Pollock, Walleye Pollock. Edinburgh: MCS, 2012. Disponível em: <http://www.fishonline.org/fish/alaska-pollockwalleye-pollock-27>. Acesso em: 01 dez. 2012. 
Avaliação do processamento a baixas temperaturas do óleo de fígado de Alaska pollock (Theragra chalcogramma)

RIBEIRO, V. A. et al.

OKADA, T.; MORRISSEY, M. T. Recovery and characterization of sardine oil extracted by $\mathrm{pH}$ adjustment. Journal of Agricultural and Food Chemistry, Davis, v. 55, p. 1808-1813, 2007.

OLIVEIRA, A. C. M.; BECHTEL, P. J. Lipid composition of Alaska pink salmon (Oncorhynchus gorbuscha) and Alaska walleye pollock (Theragra chalcogramma) by-products. Journal of Aquatic Food Product Technology, London, v. 14, n. 1, p. 73-91, 2005. http://dx.doi.org/10.1300/J030v14n01_07

OLIVEIRA, A. C. M.; BECHTEL, P. J. Lipid analysis of fillet from giant grenadier (Albatrossia pectoralis), Arrowtooth flounder (Atherestes stomias), Pacific cod (Gadus macrocephalus) and Alaska walleye pollock (Theragra chalcogramma). Journal of Muscle Foods, Hoboken, v. 17, n. 1, p. 20-33, 2006. http:// dx.doi.org/10.1111/j.1745-4573.2006.00030.x

OLIVEIRA, A. C. M.; STONE, D.; PLANTE, S.; SMILEY, S.; BECHTEL, P. J.; HARDY, R. Fish oils from Alaska seafood processing by-products: an unexploited sustainable resource for aquaculture. World Aquaculture, Baton Rouge, v. 69, p. 50-51, 2008.

PARRISH, C. C. Separation of aquatic lipid classes by chromarod thin-layer chromatography with measurement by iatroscan flame ionization detection. Canadian Journal of Fisheries and Aquatic Sciences, Ottawa, v. 44, p. 722-731, 1987. http://dx.doi. org/10.1139/f87-087

RIBEIRO, V. A. Processo de Purificação de Óleo de Pescado Utilizando Destilação Molecular. 2010. 129 f. Dissertação
(Mestrado em Engenharia e Ciência de Alimentos)-Escola de Química e Alimentos, Universidade Federal do Rio Grande, Rio Grande, 2010.

SATHIVEL, S.; PRINYAWIWATKUL, W.; KING, J. M.; GRIMM C. C.; LLOYD, S. Oil production from catfish viscera. Journal of the American Oil Chemists' Society, Urbana, v. 80, n. 4, p. 377-312, 2003.

SATHIVEL, S. Thermal and flow properties of oils from salmon heads. Journal of the American Oil Chemists' Society, Urbana, v. 82, n. 2, p. 147-152, 2005.

TIMM-HEINRICH, M.; XU X.; NIELSEN, S.; JACOBSEN, C. Application of antioxidants during short-path distillation of structured lipids. Journal of Food Lipids, Malden, v. 14, p. 244-262, 2007. http://dx.doi.org/10.1111/j.17454522.2007.00083.x

WILSON, M. T.; JUMP, C. M.; BUCHHEISTER, A. Ecology of small fishes in the Western Gulf of Alaska. II. Consumption of krill in relation to krill standing stock and the physical environment. Marine Ecology Program Series, Luhe, v. 392, p. 239-251, 2009.

WU, T. H.; BECHTEL, P. J. Quality of crude oil extracted from aging Walleye pollock (Theragra chalcogramma) byproducts. Journal of the American Oil Chemists' Society, Urbana, v. 86, p. 903-908, 2009. 